\title{
Comportamiento de variables químicas de un suelo sometido a distintos usos, departamento de Nariño, Colombia
}

\author{
Behavior of chemical variables of a soil subject to different uses, \\ department of Nariño, Colombia
}

Julio César Arteaga J.1; Jorge Fernando. Navia E..; Jesús Antonio Castillo 3

2 Ingeniero Agroforestal. Facultad de Ciencias. Universidad de Nariño, Pasto, Colombia, agroforeswamp@gmail.com.

2 Ingeniero Agrónomo. Ph.D. Facultad de Ciencias Agrícolas, Universidad de Nariño, Pasto, Colombia, jornavia@gmail.com; jornavia@yahoo.com.

3 Ingeniero Agrónomo. Ph.D. Facultad de Ciencias Agrícolas, Universidad de Nariño, Pasto, Colombia, jacf1995@gmail.com.

Citar: ARTEAGA, J.; NAVIA, J.; CASTILLO, J. 2016. Comportamiento de variables químicas de un suelo sometido a distintos usos, departamento de Nariño, Colombia. Rev. Cienc. Agr. 33(2):62-75. doi: http://dx.doi.org/10.22267/ rcia.163302.53.

Recibido: Junio 07 de $2016 . \quad$ Aceptado: Agosto 16 de 2016.

\begin{abstract}
RESUMEN
El presente estudio se realizó en un Andisol clasificado como Vitric haplustands, ubicado en el Corregimiento de Obonuco, Municipio de Pasto (Colombia). Se evaluaron algunas características químicas, en siete tipos de uso y manejo del suelo: T1: monocultivo de papa (Solanum tubersum L.), T2: pradera (Pennisetum clandestinum Hochst. ex Chiov), T3: barrera multiestrato, T4: sistema acacia - aliso, T5: banco de proteína, T6: bosque plantado (Eucaliptus globulus Labill) y T7: bosque nativo. El efecto de los tipos de uso y manejo, sobre las propiedades químicas del suelo, se evaluaron a través de $\mathrm{pH}, \% \mathrm{MO}, \% \mathrm{CO}$, $\mathrm{N}$ total, P, CIC, Ca, Mg, K, Al, Fe, Mn Cu, Zn, B y S. Los resultados fueron sometidos a Análisis de Varianza, Prueba de Comparaciones de Medias (LSD), Diferencia Mínima Significativa (DMS) y correlaciones de Persson. Los valores mostraron diferencias significativas $(\mathrm{P}<0,05)$ entre los tratamientos para los contenidos de $\mathrm{pH}$, $\% \mathrm{MO}, \% \mathrm{CO}, \mathrm{N}$ total, $\mathrm{P}, \mathrm{CIC} \mathrm{Ca}, \mathrm{Mg}, \mathrm{K}, \mathrm{Fe}$ y S, mientras que no se presentaron efectos sobre los contenidos de $\mathrm{Al}, \mathrm{Mn}, \mathrm{B}, \mathrm{Zn}$ y Cu. Los mayores valores de \%MO, \%CO, $\mathrm{N}$ total, CIC, Ca, Mg y S, fueron obtenidos con los tratamientos T3, T4 y T7. Los resultados sugieren a la materia orgánica, como la variable más sensible al efecto del manejo de los suelos.
\end{abstract}

Palabras clave: materia orgánica, usos del terreno, propiedades químicas, andisol. 


\begin{abstract}
The present study was carries out in an Andisol of the subgrup Vitric haplustands, located in the Corregimiento of Obonuco, Municipality of Pasto (Colombia). Some chemical characteristics were evaluated, in seven different soil uses and management: T1: potato monocrop (Solanum tubersum L.), T2: Kikuyo (Pennisetum clandestinum), T3: windbreak, T4: agroforestry system acacia - aliso, T5: protein blocks, T6: forest plantation (Eucaliptus globulus Labill) and T7: native forest. The effect of the soil uses and management over the chemical properties of the soils were evaluated with the variables: $\mathrm{pH} \% \mathrm{MO} \%$ $\mathrm{CO}$, total N, P, CIC, Ca, Mg, K, Al, Fe, Mn Cu, Zn, B and S. The data was analyzing using ANAVA, LSD and correlations of Pearson. The results showed significant differences $(\mathrm{P}<0,05)$ in $\mathrm{pH}$ contents, $\% \mathrm{MO}, \% \mathrm{CO}$, total N, P, CIC Ca, Mg, K, Fe and S. The higher contains of MO, CO, N, CIC, Ca, Mg and S, were obtained in the treatments T3, T4 and T7. These results allowed us to confirm the influence of M0 in the fertility of soils, being MO the variable that better detects the effect of the soil use and management.
\end{abstract}

Key words: organic matter, production systems, chemical properties, andisol.

\section{INTRODUCCIÓN}

En el departamento de Nariño durante los últimos años, las áreas dedicadas a la producción agropecuaria de los sistemas de producción convencional, se encuentran en un proceso de degradación, que se traduce en una perdida progresiva del recurso suelo y una reducción considerable en su capacidad productiva, reflejada en menores rendimientos y mayores problemas ambientales; todos estos procesos se generan por el mal manejo del suelo, problemas graves como la erosión, que han sido causados por el hombre, al no saber manejar bien sus suelos que por la acción de las fuerzas que la producen (CORPONARIÑO, 2007)

El 9.1\% del territorio nacional, tiene vocación agrícola y el $9 \%$ es de vocación ganadera, mientras un $19 \%$ tiene vocación agroforestal, el $62.5 \%$ tiene vocación forestal, de conservación y recuperación, por lo tanto, se estima que se presenta un sobre utilización de tierras en $37.2 \%$, así, alrededor del $80 \%$ de la zona andina esta afectada por la erosión, por lo tanto, uno de los mayores causantes del fenómeno es el uso del suelo por actividades agropecuarias, con tecnologías inadecuadas y sin considerar la aptitud de uso, el proceso de degradación de suelos en la región, avanza cerca de 2000 ha anuales. Para 1998, el 35\% del área de Colombia, había sido deforestada, en casi 400.000 de $1.141 .748 \mathrm{~km}^{2}$ del territorio, sin contar sabanas y páramos modificados pero no deforestado (CORPONARIÑO, 2007; CORPONARIÑO, 2012, IGAC, 2004).

Zamora (2006), quien estudió el efecto en los cambios en algunas propiedades químicas del suelo según el uso de la tierra en Venezuela, encontró, que los sistemas convencionales de producción de melón, tienden a presentar rangos inferiores en los contenidos de potasio $(\mathrm{K})$, calcio $(\mathrm{Ca})$, magnesio $(\mathrm{Mg})$, frente al sistema de producción orgánica, destacando al contenido de materia orgánica encontrado: (producción convencional $0,84 \mathrm{mg} / \mathrm{g}^{-1}$ frente a producción orgánica $3,07 \mathrm{mg} / \mathrm{g}^{-1}$ ) como responsable en los descensos encontrados, por su influencia en las propiedades del carbón orgánico $(\mathrm{CO})$ y capacidad de intercambio catiónico (CIC).

Cardona y Sadeghian (2005), encontraron que el contenido de MO fue superior en suelos de cafetales que contaban con la presencia de árboles de sombrío, en relación al manejo de monocultivo 
de café y en éste caso, el establecimiento de los árboles, mejoró las condiciones del terreno y propició un ambiente más favorable.

Del mismo modo Sacchi (2002), que el uso del suelo en suelos argentinos, así como también los procesos de erosión hídrica, han ocasionado una disminución en el carbón orgánico, donde el uso del suelo para actividades agrícolas intensivas, implica una aceleración de la mineralización, de forma tal que la materia orgánica preexistente disminuye en forma exponencial en el tiempo.

En este sentido, dado la necesidad de conocer los factores que intervienen en las propiedades del suelo, desde el punto de vista químico, se ve necesario conocer el comportamiento de las características, bajo diferentes usos de suelo, ya que la comprensión del comportamiento químico del suelo bajo este análisis, lo convierte en una instrumento primordial, que permite valorar y diagnosticar su estado (Garavito, 1984).

Por lo tanto, el objetivo de esta investigación fue el de evaluar el comportamiento de variables químicas de un suelo sometido a distintos usos en el corregimiento de Obonuco, municipio de Pasto.

\section{MATERIALES Y MÉTODOS}

Localización. El presente estudio se realizó, en la Granja Experimental del actual CORPOICA en el Corregimiento de Obonuco, Municipio de Pasto, el cual esta ubicada a $1^{\circ} 13^{\prime}$ LN y $76^{\circ} 16^{\prime}$ LO, con una altura promedio de 2720 msnm, temperatura que oscila entre 12 a $13^{\circ} \mathrm{C}$, precipitación anual promedioentre $718-703 \mathrm{~mm} /$ año y una humedad relativa de $87,5 \%$. La zona de vida, corresponde a bosque seco montano bajo, con suelos de reacción fuertemente ácida en el horizonte superior y de moderada a ligeramente ácida a mayor profundidad, alta capacidad catiónica de cambio, alta saturación de bases, altos contenidos de carbono orgánico, mediano contenido de fósforo y fertilidad química alta. Además, el estudio fue llevado en un Andisol clasificado como Vitric haplustands (IGAC, 2004).

Área experimental. El área experimental por tratamiento no fue homogénea; se realizó el muestreo en cada uno de los sistemas, sectorizando el área en tres franjas para la toma de 15 muestras en cada franja, de las cuales se obtuvo una muestra representativa por franja y 3 por sistema de producción, correspondientes a las repeticiones de cada tratamiento. Se planteo un diseño bloques completamente al azar, donde el efecto de bloque es el uso del suelo y las repeticiones las diferentes franjas en cada uso.

Descripción de los tratamientos. El tratamiento (T1), monocultivo de papa (Solanum tuberosum L.), ha sido manejado bajo un periodo de rotación de cultivos (papa - pasto), en un periodo de cinco años para cada cultivo, el área ha sido sometida a producción intensiva, con una alta aplicación de fertilizantes químicos y empleo de agroquímicos para el control de plagas y enfermedades.

El tratamiento (T2), pradera de pasto kikuyo (Pennisetum clandestinum), fue sometido a manejo rotativo papa - pasto en un lapso de 20 años. Durante los últimos siete años, esta área a permanecido bajo una unidad de manejo de pasto kikuyo con una carga animal de 1.5 animales/ha por un periodo de mes y medio en pastoreo de un día.

Tratamiento (T3), barrera multiestrato (Acacia decurrens), esta unidad fue implementada en el año 1998 con las especies acacia (Acacia decurrens), quillotocto (tecoma Stans L. Juss. ex Kunth) y mora (Rubus glaucus Benth), sin ningún manejo después de establecidas, el área adyacente a la barrera es dedicada al pastoreo, por lo que ocasionalmente se realizan ramoneos directos a la Acacia decurrens. 
El tratamiento (T4), sistema Acacia decurrens - Acacia melanoxilon y Alnus acuminata, fue implementado en el año de 1997 con las especies acacia (Acacia decurrens), acacia japonesa (Acacia melanoxilon) y aliso (Alnus acuminata). Las especies en los últimos años no han recibido ningún tipo de manejo, por lo que el área ha sido poco intervenida, no obstante la especies han respondido favorablemente a las condiciones del sito, encontrando especies que oscilan entre los 3 - $4 \mathrm{~m}$ de altura con diámetros $10-11 \mathrm{~cm}$

El tratamiento (T5), corresponde a un sistema silvopastoril banco de proteína, el cual fue establecido en el año de 1997, con una etapa inicial con las especies Acacia decurrens, Sambocus peruviana y Tecoma Stans L. En el año 2001 se realizaron investigaciones para determinar la capacidad en cuanto a producción de forraje y rebrote en las especies. En la actualidad se cuenta con bajos relictos de Acacia decurrens de buen porte, pero sin manejo y otra área se encuentra en un proceso de renovación, encontrando especies de retamo (Retama sphaerocarpa), chilca (Baccharis sp), quillotocto (Tecoma stans L. ).

El tratamiento (T6), corresponde a un bosque plantado de Eucaliptus globulus, con un tiempo de uso de 40 años; a la fecha se han realizado 3 aprovechamientos, ocasionalmente se realizan raleos selectivos de acuerdo a las necesidades de la granja.

El tratamiento (T7), bosque protector, ubicado a las faldas del Volcán Galeras, zona receptora de interés por los afluentes que alimentan la microcuenca alta de la quebrada Mijitayo. La vegetación sobresalen especies como cucharo (Clusia spp), cerote (Hesperomeles glabrata), chaquilulo (Befaria aestuans), siete cueros (Tibouchina sp), encino (Weinmania tomentosa y
Weinmania balbisiana), Pumamaque (Oreopanax $s p$ ),

Variables evaluadas. Las variables químicas evaluadas fueron: $\mathrm{pH}$ (NTC 5264), porcentaje de materia orgánica (Walkley-Black (colorimétrico) NTC 5403), porcentaje de carbono orgánico (Walkley-Black (colorimétrico) NTC 5403), nitrógeno total (con base en la materia orgánica), fósforo disponible (Bray II y Kurtz), capacidad de intercambio catiónico (acetato de amonio $1 \mathrm{~N}$ pH 7 NTC 5268), calcio, magnesio y potasio de cambio (acetato de amonio $1 \mathrm{~N} \mathrm{pH} \mathrm{7),} \mathrm{Acidez} \mathrm{de}$ cambio (extracción KCL 1N) hierro, manganeso, cobre y zinc (extracción DTPA NTC 5526), boro (extracción agua caliente NTC 5404) y azufre (extracción con fosfato monocalcico monohidratado 0.008M - NTC 5402), las cuales se realizaron en el Laboratorio de suelos e insumos agrícolas de la Universidad de Nariño (Unigarro y Carreño, 2005).

Análisis estadístico. Las diferentes variables fueron sometidas a Análisis de Varianza, Análisis de Correlaciones de Pearson; para aquellas variables, que presentaron diferencias estadísticas significativas $(\mathrm{P}<0,05)$, se realizó la Prueba de Diferencia Mínima Significativa (DMS), empleando el programa estadístico STATGRAPHICS plus 5.1.

\section{RESULTADOS Y DISCUSION}

En el ANDEVA, para las variables $\mathrm{pH}$, porcentajes de $\mathrm{MO}, \mathrm{CO}$ y $\mathrm{N}$ total, $\mathrm{P}$ disponible, CIC Ca, $\mathrm{Mg}$ y K intercambiable, Fe y $\mathrm{S}$, presentaron diferencias estadísticas significativas por el efecto de los diferentes usos y manejos, mientras que los contenidos de $\mathrm{Mn}, \mathrm{Cu}, \mathrm{Zn}$, B y Acidez cambiable no presentaron efectos significativos $(p<0,05)$. Debido a estas diferencias, a continuación se realiza el análisis de las variables que presentaron diferencias estadísticas significativas. 
Porcentajes de materia orgánica (MO), carbón orgánico (CO) y nitrógeno total $(\mathrm{N})$. Según la Prueba DMS (Tabla 1), los mayores valores promedios se presentaron en los tratamientos T3 y T4 con valores de 19,57, 11,31, 0,66, 17,90, 10,36 y $0,62 \%$, respectivamente. Se presentaron menores valores por el efecto de los diferentes manejos y usos en los tratamientos T2 y T1 con valores de 7,67, 4,44, 0,33 у T1 5,33, 3,09, 0,24\% en su orden. También los porcentajes de $\mathrm{CO}$ y N total presentan el mismo patrón de distribución con los contenidos de MO encontrados (Tabla 1).

Los resultados muestran que los tratamientos T3 y T4, en general fueron los más uniformes, y los que presentaron mejor comportamiento, debido a las características, de calidad y cantidad de biomasa que producen dichas especies T3 $(A$ decurrens), T4 (A melanoxilon, A decurrens y Alnus acuminata), lo que representa un mayor ingreso de $\mathrm{MO}$ a los sistemas y la posibilidad de transferir nitrógeno al suelo, por vía simbiótica, lo que genera una entrada adicional de $\mathrm{N}$.
Sánchez et al. (2011), reportaron que la mayor acumulación de $\mathrm{MO}$ y del $\mathrm{C}$ se registró en la superficie de suelos utilizados con piña, cítricos, eucalipto y pasturas y las menores cantidades fueron registradas en arroz, caña de azúcar y cacao. La mayor acumulación estuvo asociada con la acidez que afectó la descomposición de la MO. También, se observó disminuciones en los tratamientos T1 (monocultivo de papa) y T2 (pradera) muestran que el manejo intensivo, implica una menor incorporación de residuos de origen orgánico al sistema, lo que ocasiona una reducción paulatina en dichas características.

Crespo et al., (1998), establecen que la presencia de árboles y especialmente especies fijadoras de nitrógeno, en los sistemas productivos, incrementan la presencia de materia orgánica en el suelo, también Giraldo (1996), argumenta que la mayoría de estudios realizados en zonas tropicales, han demostrado que los árboles y arbustos leguminosas tienen la capacidad de fijar una alta cantidad de $\mathrm{N}$ hasta $150 \mathrm{~kg} / \mathrm{ha}$.

Tabla 1. DMS para características químicas estudiadas en la evaluación del manejo y uso del suelo en Obonuco, Pasto, 2008 - 2009.

\begin{tabular}{l|cccccccc}
\hline & & \multicolumn{7}{c}{ Tratamientos } \\
\cline { 2 - 8 } Parámetros & \multirow{2}{*}{ Unidad } & T1 & T2 & T3 & T4 & T5 & T6 & T7 \\
\cline { 2 - 8 } & & Papa & Kikuyo & Barrera & $\begin{array}{c}\text { Acacia } \\
\text { aliso }\end{array}$ & Proteína & Eucalipto & Bosque \\
\hline pH & & $5,46 \mathrm{~b}$ & $5,47 \mathrm{~b}$ & $5,23 \mathrm{~b}$ & $5,77 \mathrm{a}$ & $5,47 \mathrm{~b}$ & $5,36 \mathrm{~b}$ & $6,00 \mathrm{a}$ \\
Materia orgánica & & $5,33 \mathrm{~d}$ & $7,67 \mathrm{~cd}$ & $19,57 \mathrm{a}$ & $17,90 \mathrm{a}$ & $10,07 \mathrm{cb}$ & $9,03 \mathrm{c}$ & $13,43 \mathrm{~b}$ \\
Nitrógeno & $\%$ & $0,24 \mathrm{~d}$ & $0,33 \mathrm{~d}$ & $0,66 \mathrm{a}$ & $0,62 \mathrm{a}$ & $0,41 \mathrm{c}$ & $0,37 \mathrm{c}$ & $0,50 \mathrm{~b}$ \\
Carbono orgánico & & $3,09 \mathrm{~d}$ & $4,44 \mathrm{~cd}$ & $11,31 \mathrm{a}$ & $10,36 \mathrm{a}$ & $5,85 \mathrm{cb}$ & $5,24 \mathrm{c}$ & $7,78 \mathrm{~b}$ \\
CIC & & $20,73 \mathrm{c}$ & $21,20 \mathrm{c}$ & $34,60 \mathrm{a}$ & $36,27 \mathrm{a}$ & $20,87 \mathrm{c}$ & $28,73 \mathrm{~b}$ & $31,73 \mathrm{ba}$ \\
Calcio de cambio & & $9,63 \mathrm{~d}$ & $10,73 \mathrm{~d}$ & $16,60 \mathrm{~b}$ & $19,90 \mathrm{a}$ & $13,07 \mathrm{~cd}$ & $15,20 \mathrm{c}$ & $20,80 \mathrm{a}$ \\
Magnesio de Cambio & $\mathrm{Cmol}+/ \mathrm{kg}$ & $2.78 \mathrm{~d}$ & $2,62 \mathrm{~d}$ & $6,76 \mathrm{ba}$ & $9,36 \mathrm{a}$ & $3,60 \mathrm{c}$ & $3,12 \mathrm{~cd}$ & $5,00 \mathrm{~b}$ \\
Potasio de Cambio & & $1,19 \mathrm{cb}$ & $1,03 \mathrm{cb}$ & $0,97 \mathrm{~cd}$ & $0,68 \mathrm{~d}$ & $1,32 \mathrm{~b}$ & $0,92 \mathrm{~cd}$ & $1,84 \mathrm{a}$ \\
\cline { 2 - 8 } Azufre disponible & & $6,00 \mathrm{~d}$ & $7,12 \mathrm{dc}$ & $23,17 \mathrm{a}$ & $12,82 \mathrm{bc}$ & $9,06 \mathrm{c}$ & $10,29 \mathrm{c}$ & $15,00 \mathrm{~b}$ \\
Fósforo disponible & $\mathrm{mg} / \mathrm{kg}$ & $200,67 \mathrm{a}$ & $20,27 \mathrm{~b}$ & $15,97 \mathrm{~b}$ & $5,07 \mathrm{~b}$ & $16,30 \mathrm{~b}$ & $6,10 \mathrm{~b}$ & $14,33 \mathrm{~b}$ \\
Hierro & & $262,00 \mathrm{c}$ & $454,67 \mathrm{ba}$ & $505,33 \mathrm{a}$ & $393,33 \mathrm{~b}$ & $426,00 \mathrm{~b}$ & $512,67 \mathrm{a}$ & $271,33 \mathrm{c}$ \\
\hline
\end{tabular}

Promedios con letra similar no presentan diferencia estadística significativa $(\mathrm{p}<0,05)$ 
Por lo anterior, se podría deducir que los aportes en los contenidos de materia orgánica y su relación con los niveles de $\mathrm{N}$ y $\mathrm{CO}$, en los T3 y T4 presentaran mejor comportamiento, respondiendo a las características de uso y su relación con el empleo de especies leñosas leguminosas y fijadoras de nitrógeno: Acacia decurrens, Acacia melanoxilon y Alnus acuminata en el tratamiento T4 y Acacia decurrens en el T3, las cuáles pudieron constituir, el ingreso sustancial de $\mathrm{MO}, \mathrm{CO}$ y $\mathrm{N}$, en dichos sistemas.

El manejo de podas y la entrada adicional de MO por excretas, al que ha sido sometido el tratamiento T3, respondió de manera favorable en las características del suelo, ya que manifestó los porcentajes mas altos de $\mathrm{MO}, \mathrm{CO}$ y $\mathrm{N}$ total en contrástate, a los resultados manifestados en los tratamientos de manejo convencional T1 (papa) y T2 (pradera kikuyo) (Tabla 1). Ramírez (1997), en la evaluación de sistemas silvopastoriles en el Valle del Cauca, encontró que el monocultivo de pasto estrella (Cynodon plectostachyus K. Schum. Pilg.), presentó el menor contenido de $\mathrm{N}$ total y $\mathrm{MO}$, frente a los tratamientos que contaron con la presencia de árboles leguminosos leucaena (Leucaena leucocephala (Lam.) de Wit) y algarrobo (Prosopis juliflora), para las profundidades de $0-10 \mathrm{y}$ 10-20 cm, debido al aporte de material orgánico en forma de hojarasca y residuos de poda que se generan en el estrato arbóreo, de igual manera la sombra generada por estas especies, hace que la mineralización de la reserva orgánica del suelo sea más lenta que la ocurrida en praderas a plena exposición del sol.

Giraldo (1996), reporto en sistemas silvopastoriles de Acacia decurrens en Antioquia, los incrementos sustanciales de $\mathrm{MO}$ y N; debido a la alta producción de biomasa (4.0 ton/ha/año) que generan estas especies, de igual manera el alto contenido proteico de la misma, lo cual, fomenta una mayor actividad biológica facilitando su descomposición.
De las Salas (1987), afirma que en los ecosistemas boscosos naturales, los contenidos de materia orgánica, tienden a ser altos, de acuerdo a una dinámica interna de ciclo cerrado por lo que reduce perdidas significativas de $\mathrm{MO}$, en el sistema, a diferencia de sistemas productivos intensivos convencionales, que responden a una mayor taza de mineralización por efecto de manejo. Si bien los valores de $\mathrm{MO} \mathrm{CO}$ y $\mathrm{N}$ total encontrados en el estudio fueron altos para el tratamientos $\mathrm{T} 7$, los resultados encontrados presentaron diferencias con los tratamientos T3 (multiestrato Acacia, quillotocto y mora) y T4 (Acacia decurrens; Acacia melanoxilum y Alnus acuminota) (Tabla 1), es probable que las reducciones encontradas en el tratamiento T7 (bosque protector), respondan a las condiciones agroecológicas del sitio, ya que al pertenecer a una zona cercana a los 3100 msnm, condiciones en donde predominan bajas temperaturas y alta humedad, son factores que afectan los procesos de mineralización y facilita el lavado del material orgánico por erosión hídrica (Fassbender, 1983), situación que pudo haber influido con respecto a los tratamientos T3 (multiestrato Acacia, quillotocto y mora) y T4 (Acacia decurrens; Acacia melanoxilum y Alnus acuminota) .

A diferencia de estos resultados Geissert y Ibáñez (2000), en su trabajo de calidad y ambiente físico-químico de los suelos, encontraron diferencias significativas entre el bosque y los sistemas cafeteros, el contenido de $\mathrm{MO}$ (23,9 - 27,6g.kg- $\left.{ }^{1}\right), \mathrm{CO}\left(41,3\right.$ - 47,7g.kg- $\left.{ }^{1}\right)$ y $\mathrm{N}$ total $\left(4,0-4,4 \mathrm{~g} \cdot \mathrm{kg}^{-1}\right)$ en el bosque fue alto con respecto a las unidades cafeteras MO (13,3 - 17,4g.kg- $\left.{ }^{1}\right) \mathrm{CO}\left(23,1\right.$ - 30,1g.kg- $\left.{ }^{1}\right)$ y $\mathrm{N}$ total $\left(2,74\right.$ - 1,02g.kg- $\left.{ }^{1}\right)$, debido a una mayor taza de aporte de residuos orgánicos que generan los ecosistemas boscosos. Por lo anteriormente mencionado, la respuesta en los porcentajes de $\mathrm{MO}, \mathrm{CO}$ y $\mathrm{N}$ total por la introducción de especies fijadoras de $\mathrm{N}$, en los tratamientos $\mathrm{T} 3$ (multiestrato Acacia, quillotocto y mora) y T4 
(Acacia decurrens; Acacia melanoxilum y Alnus acuminota), se convierten en un factor relevante en los resultados encontrados.

Los resultados permiten considerar, que el tipo de vegetación influye en los porcentajes de $\mathrm{MO}, \mathrm{CO}$ y N total en el suelo. Los porcentajes encontrados en el tratamiento T6 responden a estos factores, según Fassbender y Bornemiza (1994) y De la Salas (1987), especies con bajas proporciones de proteína, alta relación $\mathrm{C} / \mathrm{N}$, afectan los procesos de mineralización. Manzanares et al., (2004), reportaron que en plantaciones forestales de Eucalipto globulus y Pinus radiata por efecto de la sustitución del bosque nativo sobre el horizonte orgánico en Concepción (Chile), los contenidos de materia orgánica se redujeron en un $40-50 \%$, valor explicado por la relación $\mathrm{C} / \mathrm{N}$ alta, que genera la cubierta vegetal de estas especies, también, Ceccon (1999), argumenta que la alta relación $\mathrm{C} / \mathrm{N}$ que genera el mantillo del eucalipto, presenta un velocidad de descomposición lenta, generando una reducida mineralización de la materia orgánica suministrada por el sistema, razón por la cual se puede inferir que las diferencias encontradas en los porcentajes en el tratamiento T6 (bosque de eucalipto) en comparación a los tratamientos T3 (multiestrato Acacia, quillotocto y mora) y T4 (Acacia decurrens; Acacia melanoxilum y Alnus acuminota), y T7 (bosque protector), responde a la baja descomposición (Tabla 1).

Los tratamientos T1 (papa) y T2 (pradera kikuyo), presentaron los menores valores promedios, en los porcentajes de $\mathrm{MO}, \mathrm{CO}$ y $\mathrm{N}$ total (Tabla 1). El monocultivo intensivo genera una disminución progresiva de estos elementos, debido a que existe una disminución en la incorporación de residuos de origen orgánico al sistema, por lo que el efecto del uso y manejo generan cambios significativos en los porcentajes en mención; Rondón et al (1998), mencionan, en el efecto de cambios en el uso del suelo so- bre los almacenamientos de carbono orgánico en áreas del páramo en Cauca - Colombia; donde, los niveles en las capas superficiales de suelos bajo cultivos, presentaron significativamente menores tazas de carbón orgánico $(5-4 \%)$, debido a una menor incorporación de materia orgánica en el sistema, frente a los contenidos altos de $\mathrm{CO}$ en la zonas de bosque de páramo no intervenido (10 - 20\%).

Las disminuciones reportadas en los tratamientos T1 (papa) y T2 (pradera kikuyo), son consistentes con los efectos esperados, debido que al aumentar la tasa de mineralización de la materia orgánica del suelo, por el efecto de labranza y preparación del suelo para el establecimiento y manejo del cultivo, donde se estima que se incorporan nuevos suelos a la agricultura hasta establecer sistemas intensivos de cultivo, se producen pérdidas de $\mathrm{CO}$ que fluctúan entre 30 y $50 \%$ del nivel inicial (Fassbender, 1983 y Martínez et al., 2008).

De manera general, las diferencias encontradas, responden a las características de la vegetación y la adaptación de las mismas a las condiciones del sitio (factores climáticos y edafológicos, entre otros); también, los cambios de uso de suelo disminuyen o aumentan la mineralización del C, el nitrógeno de la biomasa microbiana y el carbono de la biomasa microbiana (Cruz et al., 2012), siendo esto un factor determinante en cuanto a porcentajes de $\mathrm{MO}$ y patrones de acumulación de $\mathrm{N}$ total y $\mathrm{CO}$ en el suelo (Tamhane, 1979, De las Salas, 1987 y Fassbender, 1983); donde, se resalta la importancia ecológica que poseen el establecimiento de barreras vivas en las características del suelo.

El N y CO alcanzaron una correlación positiva y significativa de $(\mathrm{r}=0,99)$ con la MO (Tabla 2 ), lo que demuestra que las variaciones en los contenidos de $\mathrm{N}$ y CO, están determinadas por la dinámica de la materia orgánica en el suelo (De Las Salas, 1987; Fassbender, 1993). 


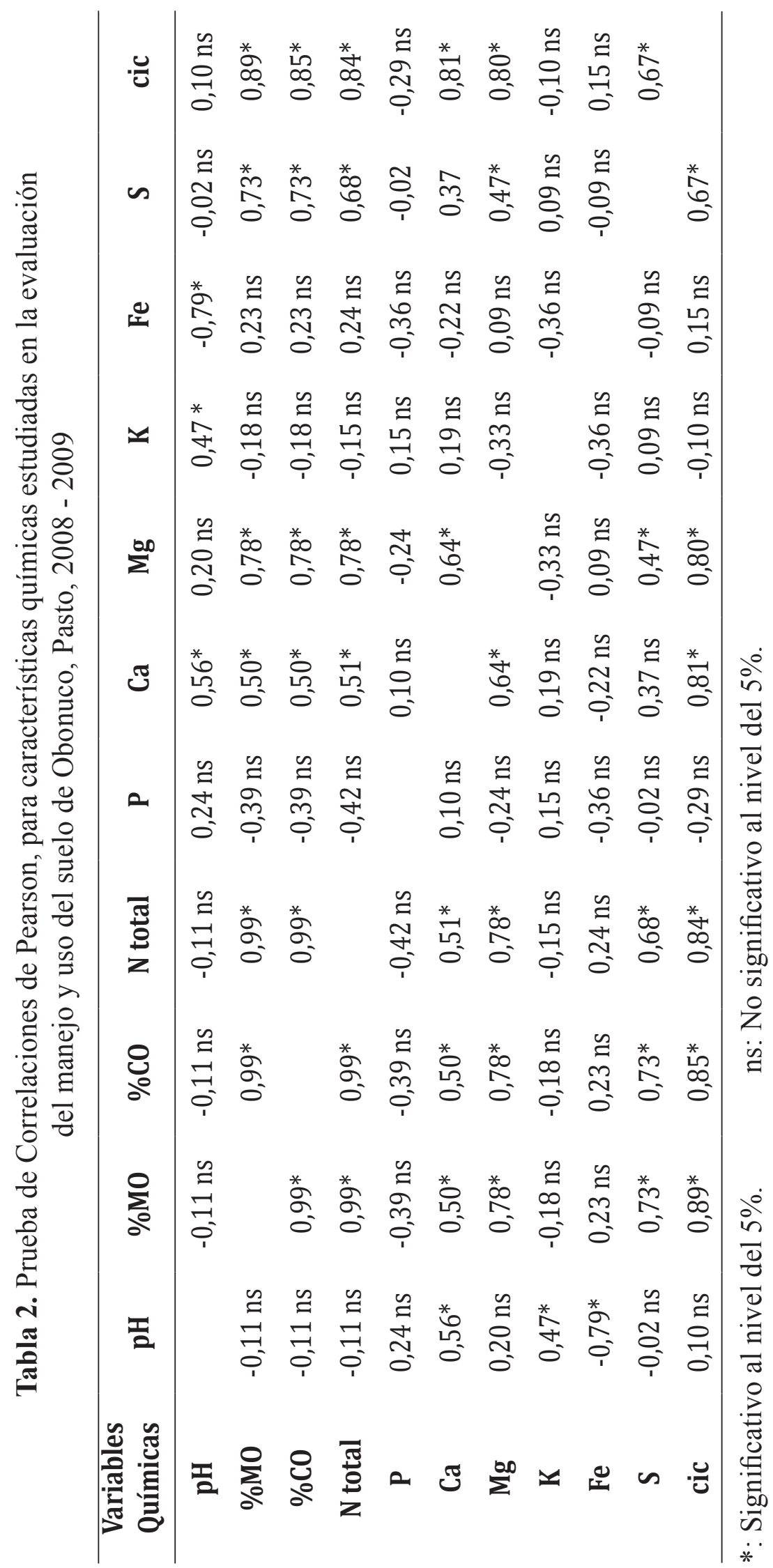


Según Fassbender y Bornemisza (1994), entre el $95-98 \%$ del nitrógeno total esta asociado a sustancias orgánicas, convirtiendo a la materia orgánica como fuente primaria de este elemento, mientras que Martínez et al. (2008) argumentan que el carbón orgánico, representa el $58 \%$ de la materia orgánica.

El estudio encontró, que los contenidos de $\mathrm{CO}$, se relacionaron con la $\mathrm{CIC}(\mathrm{r}=0,85) \mathrm{Ca}(\mathrm{r}=0,50)$ $\mathrm{Mg}(\mathrm{r}=0,78)$ y $\mathrm{N}(\mathrm{r}=0,99)$ (Tabla 2$)$, vinculando al CO con la cantidad y disponibilidad de nutrientes del suelo, De la Salas, (1987) sustenta que el CO habilita las propiedades del suelo relacionadas con el rendimiento sostenido de los cultivos al aportar elementos a los sistemas agrícolas. Sacchi (2002), en la evaluación de los cambios en las propiedades físico químicas por procesos de degradación en Argentina, señalan que las propiedades químicas tales como la materia orgánica y carbón orgánico son las variables que mejor detectan la degradación de los suelos.

Azufre (S). El tratamiento T3 (multiestrato Acacia, quillotocto y mora- $23,17 \mathrm{mg} / \mathrm{kg}$ ), presenta el mayor promedio por efecto de uso y manejo del suelo, el cual presenta diferencias estadísticas significativas con los tratamientos T2 (pradera kikuyo- $7,12 \mathrm{mg} / \mathrm{kg})$ y T1 $(6,00 \mathrm{mg} /$ $\mathrm{kg}$ ), los que presentaron los menores valores promedios en el estudio (Tabla 1).

Las fuentes orgánicas de los suelos, finalmente son las encargadas de promover las variaciones en los contenidos de azufre (De la Salas 1987; Fassbender y Boernemiza, 1994), por lo que la mineralización de dicho elemento, depende de los factores generales que regulan la acumulación y mineralización de la materia orgánica. El azufre presentó correlación positiva y significativa con el porcentaje de MO $(r=0,73)$ (Tabla 2 ), por lo que se puede afirmar, que los resultados fueron significativos, debido a que los procesos dinámicos del azufre, están influenciados por las variaciones de la materia orgánica.
Calcio (Ca) y magnesio (Mg). Según la prueba DMS (Tabla 1) los mayores valores promedios de $\mathrm{Ca}$ se presentaron en los tratamientos T7 (bosque protector) y T4 (Acacia decurrens; Acacia melanoxilum y Alnus acuminota) con valores de $20,80 \mathrm{cmol} / \mathrm{kg}$ y $19,90 \mathrm{cmol} / \mathrm{kg}$ y en Mg en los tratamientos tratamientos T4 (Acacia decurrens; Acacia melanoxilum y Alnus acuminota) y $\mathrm{T} 3$ (multiestrato Acacia, quillotocto $\mathrm{y}$ mora), con valores de $9,36 \mathrm{cmol} / \mathrm{kg}$ y $8,76 \mathrm{cmol} /$ $\mathrm{kg}$. Los tratamientos en mención, muestran diferencias significativas por el efecto de uso y manejo con relación a los tratamientos T2 (pradera kikuyo) y T1 (papa), los cuales presentaron los menores rangos en el estudio, con valores promedios de $10,73 \mathrm{cmol} / \mathrm{kg}, 9,63 \mathrm{cmol} / \mathrm{kg}$ y 2,78 $\mathrm{cmol} / \mathrm{kg}, 2,62 \mathrm{cmol} / \mathrm{kg}$, respectivamente.

La distribución de estos elementos, en general responden a la relación con los patrones de materia orgánica localizadas en la evaluación. El calcio mostró correlaciones positivas y significativas con la MO $(\mathrm{r}=0,50), \mathrm{N}$ total $(\mathrm{r}=0,51)$ y $\mathrm{CO}(\mathrm{r}=0,50)$ y el magnesio con la $\mathrm{MO}(\mathrm{r}=0,78)$, $\mathrm{N}$ total $(\mathrm{r}=0,78), \mathrm{CO}(\mathrm{r}=0,78)$ y $\mathrm{S}(\mathrm{r}=0,47)(\mathrm{Ta}-$ bla 2); lo que es explicable, debido a que en el proceso de mineralización que conlleva a la disociación de $\mathrm{NO}_{3}^{-}, \mathrm{SO}_{4}=$ y coloides húmicos, cuyas cargas resultantes son electronegativas, son las que proporcionan el incremento sustancial en la capacidad de cambio, brindando una mayor posibilidad de retener $\mathrm{Ca}$ y $\mathrm{Mg}$ en el suelo (Fassbender y Bornemisza, 1994, De las Salas, 1987).

Similares resultados fueron reportados por Zamora (2006), en el efecto de dos sistemas de producción convencional y orgánica de melón. Para la producción orgánica, niveles de $\mathrm{Ca}$ y $\mathrm{Mg}$ fueron de $0,93 \mathrm{cmol} / \mathrm{kg}, 1,02 \mathrm{cmol} / \mathrm{kg}$, frente a la producción convencional $\mathrm{Ca}$ y $\mathrm{Mg}$ con valores de $0,43 \mathrm{cmol} / \mathrm{kg}, 0,98 \mathrm{cmol} / \mathrm{kg}$. La incorporación y la paulatina mineralización de los residuos orgánicos, generan aumentos en la capacidad de cambio, de igual manera, la mineralización 
de una parte estimable de la materia orgánica, restituye una fracción de calcio y magnesio extraído al suelo.

Las variaciones encontradas entre tratamientos, señalan, que los sistemas productivos que involucran una menor taza de incorporación de residuos orgánicos, generan una reducción progresiva de $\mathrm{Ca}$ y $\mathrm{Mg}$. Los descensos encontrados, en los tratamientos T1 (papa) y T2 (pradera kikuyo) (Tabla 1), de acuerdo al uso y manejo, presentan una menor incorporación de materiales de origen orgánico y se convierte en el factor más relevante, en las disminuciones localizadas en el estudio.

CIC. Los mayores valores promedios por efecto de uso y manejo del suelo se obtuvieron en los tratamientos T4 (Acacia decurrens; Acacia melanoxilum y Alnus acuminota) y $\mathrm{T} 3$ (multiestrato Acacia, quillotocto y mora), y T7 (bosque protector), con valores de 36,27, 34,60 y $31,73 \mathrm{cmol} / \mathrm{kg}$, en su orden. Se obtuvieron diferencias estadísticas significativas en los tratamientos T2 (pradera kikuyo), T5 (silvopastoril banco proteína) y T1 (papa), con valores de $21,20 \mathrm{cmol} / \mathrm{kg}, 20,87$ y $20,73 \mathrm{cmol} / \mathrm{kg}$ respectivamente, los cuales manifestaron promedios más bajos (Tabla 1).

La capacidad de cambio encontrada en las unidades de manejo es alta $(>20 \mathrm{cmol} / \mathrm{kg})$, en razón a que los suelos en estudio responden a una mineralogía de influencia volcánica, donde se destaca la presencia de alófana, material que influye en los fenómenos de absorción (Fassbender y Bornemizsa, 1994). No obstante, el estudió encontró una correlación positiva y significativa $(\mathrm{r}=0,89)$, con la materia orgánica y carbón orgánico $(\mathrm{r}=0,85)$ (Tabla 2), de ahí que las diferencias localizadas, comprometen a los contenidos de MO encontrados entre los tratamientos, debido a la intervención de la $\mathrm{MO}$, la cual contribuye a promover dicha capacidad en el suelo.
Sobre el particular Fassbender (1983), argumenta que la CIC permanente, proviene de la fracción arcilla, mientras que la CIC depende de las sustancias húmicas por la disociación de los grupos carboxílicos y fenólicos, y pueden llegar a tener una CIC que superan los $150 \mathrm{cmol} /$ $\mathrm{kg}$, lo cual explica el incremento encontrado en la capacidad de intercambio catiónico (CIC), en los tratamientos T4 (Acacia decurrens; Acacia melanoxilum y Alnus acuminota- 36,27 $\mathrm{cmol} / \mathrm{kg}$ )) y T3 (multiestrato Acacia, quillotocto y mora- $34,60 \mathrm{cmol} / \mathrm{kg}$ ). De la misma forma, dichos resultados declinaron en los sistemas de manejo convencional T2 (pradera kikuyo$21,20 \mathrm{cmol} / \mathrm{kg}$ ) y T1 (papa- $20,73 \mathrm{cmol} / \mathrm{kg}$ ).

Es evidente, que los tratamientos continuaron con la misma tendencia que los valores encontrados, a excepción del tratamiento T5 (silvopastoril banco proteína- $20,87 \mathrm{cmol} / \mathrm{kg}$ ), el cual tiene similar comportamiento a los tratamientos T2 (pradera kikuyo) y T1 (papa). No se descarta la posible influencia de arcillas alófanas en el resultado. De manera general, los coloides orgánicos son altamente dependientes del uso y manejo.

Resultados similares fueron reportados por Zamora (2006), quien encontró, que la disminución en los contenidos de materia orgánica, en un sistema de manejo convencional de melón en Venezuela, redujo la CIC en $(11,76 \mathrm{cmol} / \mathrm{kg})$, en producción de (Cucumis melo L.), convencional, en comparación, al sistema de manejo de melón con abonos orgánicos $(16,89 \mathrm{cmol} /$ $\mathrm{kg}$ ), demostrando el efecto de la materia orgánicas en las propiedades del suelo.

Potasio (k). Según la Prueba de DMS (Tabla 1), el mayor promedio de K correspondió al tratamiento T7 (bosque protector- 1,84 cmol/ $\mathrm{kg}$ ), el cual presenta diferencias estadísticas significativas con los tratamientos T3, T6 y T4 con valores promedio de $0,97,0,92$ y $0,68 \mathrm{cmol} / \mathrm{kg}$ respectivamente. 
Tamhane (1979), manifiesta que la dinámica del $\mathrm{K}$ en el suelo se encuentra relacionado con los contenidos de $\mathrm{MO}$ en respuesta al ciclaje de nutrientes que se genera por efecto de la mineralización; contrario a esto último, el estudio no encontró relación alguna con los patrones de $\mathrm{MO}$ y $\mathrm{CO}$; es presumible que los efectos encontrados y las cantidades de potasios encontrados, se encuentran ligadas a la presencia de minerales primarios, que son frecuentes en suelos de origen volcánico, por lo que se convierte en un factor determinante en los resultados encontrados (Fassbender y Bornemisza, 1994 y IGAC 2004).

Razz y Clavero (2006), en su trabajo sobre cambios en las características químicas de suelos, en un banco de proteína de Leucaena leucocephala (Lam.) y en un monocultivo de Brachiaria brizantha, con respecto a los contenidos de $\mathrm{K}$, reportaron que el valor de $\mathrm{K}$, fue superior en el uso de Leucaena leucocephala $(0,9 \mathrm{me} / 100 \mathrm{~g})$, en relación al obtenido en el monocultivo de Brachiaria brizantha (0,5 me/100 g); de acuerdo a estos datos, los autores afirman que la presencia de árboles en los potreros, incrementa la presencia de materia orgánica y mejora la fertilidad de los suelos.

Es importante mencionar, que este estudio, no encontró relación alguna con los patrones de materia orgánica; no obstante, se presenta correlación positiva y significativa con el $\mathrm{pH}$ $(r=0,47)$. Las diferencias entre tratamientos, responden a la relación del $\mathrm{K}$ con los patrones de $\mathrm{pH}$ encontrados (Tabla 2). El pH ejerce un papel importante en la determinación de la cantidad y tipo de carga en los suelos, al aumentar el $\mathrm{pH}$ del suelo, más iones $\mathrm{OH}^{-}$se vuelven disponibles para crear sitios de cargas negativas, exhibiendo una mayor saturación de bases en el suelo (Garavito, 1984; Tamhane, 1979; Fassbender y Bornemisza, 1994).
Fósforo (P). El análisis mostró que el tratamiento T1 (papa), presentó el mayor valor de $\mathrm{P}$ disponible en el estudio (Tabla 1). Es factible que exista una mayor disponibilidad de P disponible en la solución del suelo, ya que en el momento del muestro, el lote fue sujeto a fertilización química.

Si bien los demás tratamientos no presentaron diferencias estadísticas significativas, se puede observar que los tratamientos T6 (bosque de eucalipto) y T4 (Acacia decurrens; Acacia melanoxilum y Alnus acuminota) con valores de 6,10 y $5,07 \mathrm{mg} / \mathrm{kg}$ presentan los promedios mas bajos en el estudio (Tabla 1). Es presumible, que estos valores responden a una mayor capacidad extractiva de este elemento por parte de: Alnus acuminata, Acacia melanoxilon y Eucaliptus globulus. Segura et al., (2005), encontraron plantaciones de aliso, la extracción de $\mathrm{P}$ oscilaba en $193,6-879 \mathrm{~kg} / \mathrm{ha} / \mathrm{P}$ en plantación de siete años.

Soalleiro et al. (2004), reportan el efecto de la silvicultura en la extracción de nutrientes a lo largo del turno en plantaciones de tres especies de crecimiento rápido, encontrando que las plantaciones de Eucaliptus globulus extraen entre 192 a $1537,6 \mathrm{~kg} / \mathrm{ha} / \mathrm{P}$ valores altamente dependientes de la edad de la plantación, debido a que Alnus acuminata, Acacia melanoxilon y Eucaliptus globulus, son consideradas de alto valor maderable. De acuerdo a lo anterior, Tokura, 1996 y Soalleiro et al., 2004, argumentan que para lograr ese status de madera, se requieren elevados niveles de energía y por eso el $\mathrm{P}$ provee el mecanismo de almacenamiento y transformación de esta energía en forma de ATP, lo que facilita funciones como la desnaturalización del $\mathrm{CO}_{2}$ en $\mathrm{C}$, representando una mayor lignificación de sus estructuras vegetativas.

Por lo tanto, es factible que las disminuciones encontradas en los tratamientos T4 (Acacia decurrens; Acacia melanoxilum y Alnus acuminota) 
y T6 (bosque eucalipto), respondan a una mayor necesidad fisiológica de éste elemento por parte de estas plantaciones. Según Borges et al. (2012), en su estudio sobre características físico - químicas del suelo y su asociación con macro elementos, concluyen que las características de relieve y $\mathrm{pH}$ actúan como factores predisponentes en la disponibilidad de algunos minerales y MO, y que el fósforo fue el elemento mayormente carente en usos del suelo con pastoreo de bovinos.

pH. Los tratamientos T7 (bosque protector) y T4 (Acacia decurrens; Acacia melanoxilum y Alnus acuminota) presentaron los mayores valores promedios, con valores de 6,00 y 5,77, presentando diferencias estadísticas significativas con los tratamientos T2 (pradera kikuyo), T5 Silvopastoril banco proteína), T1 (papa), T6 (bosque eucalipto), y T3 (multiestrato Acacia, quillotocto y mora), con valores promedio de $5,47,5,47,5,46,5,36$ y 5,23 respectivamente (Tabla 1).

Los resultados, muestran que los tratamientos T7 (bosque protector) y T4 (Acacia decurrens; Acacia melanoxilum y Alnus acuminota), presentan una acidez media ( $\mathrm{pH}$ 5,5 - 6,0) en virtud, a que en la reacción del suelo responde a fenómenos propios de la mineralización de la materia orgánica. Según (De las Salas, 1987; Martínez et al, 2008), el N contenido en los materiales vegetales, provoca un aumento inicial de $\mathrm{pH}$ asociado a formación de $\mathrm{NH}_{4}^{+}$, lo que resulta evidente en el aumento de $\mathrm{pH}$, en los suelos de los tratamientos mencionados, De las salas (1987), argumenta que los ecosistemas forestales tienden a generar mayores acumulaciones de materia orgánica lo que facilita una mayor intervención sobre la reacción del suelo, dichos valores pueden variar por las características de la vegetación.

En general, los tratamientos T2 (pradera kikuyo), T5 Silvopastoril banco proteína), T1 (papa), T6 (bosque eucalipto), y T3 (multiestra- to Acacia, quillotocto y mora), presentaron un comportamiento similar (Tabla 1), encontrando al tratamiento T2 con el rango más alto $(5,47)$ y el T3 con el más bajo $(5,23)$. La reacción del suelo encontrada se caracteriza por ser fuertemente acida $(5,0$ - 5,5), aunque el estudio encontró baja saturación de aluminio cambiable, la acidez encontrada puede obedecer a los grupos funcionales de la materia orgánica carboxílicos y fenólicos que se comportan como ácidos $\mathrm{y}$ tienden a disminuir el $\mathrm{pH}$ del suelo (Fassbender y Bornemiza, 1994).

La acidez, se relacionó con la bases intercambiables principalmente con el calcio $(\mathrm{r}=0,56)$ y el potasio $(r=0,47)$ (Tabla 2$)$. Estos valores, indican que en presencia de niveles altos de $\mathrm{pH}$, se aumenta la absorción de bases cambiables en la reacción del suelo, minimizando pérdida de nutrientes por fenómenos de lixiviación (Tamhane, 1979). Similares resultados fueron reportados por Geissert y Ibáñez (2000), en su trabajo de calidad y ambiente físico-químico de los suelos, los suelos cafeteros y forestales. La acidez, presentó diferencias significativas entre ambos, relacionando la acidez con la saturación de bases intercambiables $(r=0,71, p \leq 0,05)$ calcio, potasio y magnesio.

Hierro (Fe). Según prueba DMS (Tabla 1), los tratamientos T6 (bosque eucalipto), T3 multiestrato Acacia, quillotocto y mora) y T2 (pradera kikuyo) presentan los mayores rangos, con valores promedios de 512,67, 505,33 y $454,67 \mathrm{mg} / \mathrm{kg}$, los cuales presentan diferencias estadísticas significativas con los tratamientos T7 (bosque protector-271,33mg/kg) y T1 ( papa$262,00 \mathrm{mg} / \mathrm{kg}$ ).

Se destaca igualmente alta presencia de hierro en los tratamientos ( $>50 \mathrm{mg} / \mathrm{kg}$ ), debido a la naturaleza de los suelos evaluados, de mineralogía propia de zonas de influencia volcánica, por la participación de goethita, hematita, limonita y otros óxidos e hidróxidos de hierro, 
producto de la meteorización de rocas ígneas (Fassbender y Bornemisza, 1994). Las diferencias encontradas comprometan a la mineralogía de los suelos de estudio (influencia volcánica); Galindo (2005), reporto en la evaluación físicoquímica de la materia orgánica en un suelo de tradición papera; altos contenidos de $\mathrm{Fe}$ en un cultivo de papa (434 ppm) similares a los del bosque (478 ppm), respondiendo a factores relacionados por la alta influencia de la mineralogía de los suelos de estudio (andisoles). Fassbender (1983); argumenta que la materia orgánica puede actuar como solubilizador de material mineral incrementando de esta manera la disponibilidad de micronutrientes para las plantas; contrario a esto el estudio no encontró relación con los contenidos de hierro y materia orgánica.

Por otra parte, los resultados siguieren la relación entre los contenidos de hierro con los niveles de $\mathrm{pH}$ la cual responde a una correlación de $(r=-0,79)$, debido a que la solubilidad del hierro incrementa a $\mathrm{pH}$ menores y se reduce cuando se manifiestan aumentos progresivos (Tabla 2), debido a su precipitación en forma de hidróxido férrico $\mathrm{Fe}(\mathrm{OH})_{3}$ (Garavito, 1984, Fassbender, 1993, y Fassbender y Bornemisza, 1994), es presumible que las diferencias encontradas respondan a este fenómeno.

\section{CONCLUSIONES}

El manejo convencional T1 monocultivo de papa (Solanum tubersum L.) y T2: pradera (Pennisetum clandestinum) presentaron disminución en el porcentaje de $\mathrm{CO}, \mathrm{MO}, \mathrm{N}$ total y bases intercambiables.

Los tratamientos T3 (barrera multiestrato) y T4 (sistema acacia - aliso) generan las mejores condiciones del suelo por las respuestas en MO y fertilidad.

El establecimiento de especies arbóreas fijadoras de nitrógeno en los sistemas de producción mejoran significativamente, las condiciones químicas de los suelos, constituyéndose una alternativa en suelos deficientes en nutrientes para mejorar la fertilidad.

\section{AGRADECIMIENTOS}

A la Federación Colombiana de Productores de Papa (FEDEPAPA) y a la Asociación Hortofrutícola de Colombia por el financiamiento de esta investigación.

\section{REFERENCIAS BIBLIOGRÁFICAS}

BORGES, J.; BARRIOS, M.; SANDOVAL, E.; BASTARDO, Y.; OSWALDO, M. 2012. Características físico-químicas del suelo y su asociación con macroelementos en áreas destinadas a pastoreo en el estado Yaracuy. Bioagro. 24(2):121 - 126.

CARDONA, A.; SADEGHIAN, J. 2005. Evaluación de propiedades físicas y químicas de suelos establecidos con café bajo sombra y a plena exposición solar. Revista CENICAFE 56(4):348 - 364.

CECCON, E. 1999. Aspectos ambientales referentes al establecimiento de plantaciones de eucalipto de gran escala en áreas tropicales. Revista Interciencia. 24 (5):61 - 67

CORPONARIÑO. CORPORACIÓN AUTÓNOMA REGIONAL DE NARIÑO. 2007. Plan de acción trienal (PAT) 2007 - 2009. En: http://corponarino.gov.co/expedientes/planeacion/informe_gestion2008/informegestionfinal2008.pdf; consulta: mayo, 2015.

CORPONARIÑO. CORPORACIÓN AUTÓNOMA REGIONAL DE NARIÑO. 2012. Plan de gestión ambiental regional. 2002 - 2012.En: http://corponarino.gov.co/ expedientes/planeacion/pgar20152032/diagnosticoPGAR20152032-parte1.pdf; consulta: mayo, 2015.

CRESPO, G.; RODRÍGUEZ, I.; SÁNCHEZ, R.; FRAGA, S. 1998. Influencia de Albizia lebbeck y leucaena leucocephala en indicadores de suelo, el pasto y los animales en sistemas silvopastoriles. En: CIPAV, http://www.cipav.org. co/redagrofor/memoria99/P-Crespo.htm; consulta: mayo, 2015.

CRUZ, A.; CRUZ, E.; AGUILERA, L.; NORMAN, H. T.; FRANCO, S.; NAVA, G.; DENDOOVEN, L.; REYES, B. G. 2012. La biomasa microbiana en suelos de montaña con diferentes usos: un estudio de laboratorio. Terra Latinoamericana. 30(3):221 - 228. 
DE LAS SALAS, G. 1987. Suelos y ecosistemas forestales con énfasis en America tropical. Instituto Interamericano de Cooperación para la Agricultura. IICA, San José Costa Rica. $447 \mathrm{p}$.

FASSBENDER H. 1983. Suelos y sistemas de producción agroforestales. CATIE. Turrialba, Costa Rica. 250 p.

FASSBENDER. H. 1993. Modelos edafológicos de sistemas agroforestales. Segunda edición. CATIE. Turrialba, Costa Rica. 487 p.

FASSBENDER, H.; BORNEMISZA, E. 1994. Química de suelos con énfasis en suelos de América Tropical. IICA, Costa Rica. 418 p.

GALINDO, D. 2005. Evaluación físico - química de la materia orgánica en un suelo de tradición papera. Revista de Agronomía. 13(1):37 - 58.

GARAVITO, F. 1984. Propiedades químicas de los suelos. Instituto Geográfico Agustín Codazzi, Bogota D.C. 560 p.

GEISSERT, D.; IBÁÑEZ, A. 2000. Calidad y ambiente fisico-químico de los suelos. Revista Agrociencia. 36(5):605 -620 .

GIRALDO, L. 1996. Evaluación del potencial multipropósito en dos especies de Acacia para el desarrollo de sistemas silvopastoriles en clima frío. P. 9-12. En: Memoria V. Congreso Colombiano de Ciencias Pecuarias.

IGAC. INSTITUTO GEOGRAFICO AGUSTIN CODAZZI. 2004. Estudio general de suelos y zonificación de tierras departamento de Nariño. ftp://gisweb.ciat.cgiar.org/ DAPA/users/apantoja/london/Colombia/Suelos/00 shape_suelos/PROYECTO_DNP/MEMORIAS_SUELOS OFICIALES/NARI\%C3\%910/Cap\%201.pdf

MANZANARES, N.; TORREZ, R.; NAVARRO, C. 2004. Efecto de la sustitución del bosque nativo por plantaciones de pino y eucalipto sobre el horizonte orgánico en concepción (Chile). Revista ITEA. 100 (2):96 - 109.

MARTÍNEZ, H.; FUENTES, E.; ACEVEDO H. 2008. Carbono orgánico y propiedades del suelo. R. Suelo y Nutrición Vegetal. 8 (1):68 - 96.

RAMÍREZ, H. 1997. Evaluación de dos sistemas silvopastoril es integrados por Cynodon plectostachyus, Leucaena leucocephala y Prosopis juliflora. En: Seminario Internacional de Sistemas Sostenibles de Producción Agropecuaria. CIPAV. Cali.

RAZZ, A.; CLAVERO, J. 2006. Cambios en las características químicas de suelos en un banco de Leucaena leucocephala y en un monocultivo de Brachiaria brizantha. Revista de la Facultad de Ciencias Agrícolas. 15(1):326 - 331.

RONDÓN, M.; AMÉZQUITA, E.; CHÁVEZ, F. 1998. Efecto de cambios en el uso del suelo sobre los almacenamientos de carbono y flujos de gases de efecto invernadero en áreas del páramo de las ánimas, cauca, Colombia. p.133-141. En: Memorias XIV Congreso Venezolano de la ciencia del suelo.

SACCHI, G. 2002. Evaluación de los cambios en las propiedades físicas y químicas de un argiustol udico por procesos de degradación. Revista Agrociencia. 5(2):37 - 46.

SÁNCHEZ, R.; RAMOS, R.; GEISSEN, V.; MENDOZA, J. D. DE LA CRUZ, E.; SALCEDO, E.; PALMA, D. 2011. Contenido de carbono en suelos con diferentes usos agropecuarios en el trópico mexicano. Terra Latinoamericana. 29(2):211 - 219 .

SEGURA, M.; CASTILLO, A.; ALVARADO, A.; BLANCO F. 2005. Extracción de nutrimentos en plantaciones de jaúl (Alnus acuminata) en la cuenca del río Virilla, Costa Rica. Revista Agronomia Costarricense. 29(2):109 - 120.

SOALLEIRO, R.; BALBOA, M.; GONZÁLEZ, A. 2004. Efecto de la silvicultura en la extracción de nutrientes largo del turno en plantaciones de tres especies de crecimiento rápido en el norte de España. Invest Agrar. 114 - 126 p.

TAMHANE. H. 1979. Suelos su química y fertilidad en zonas tropicales. Mexico, Diana. 483p.

TOKURA, Y. 1996. Árboles nativos del Valle del Cauca. Cali, Colombia: CVC. FUNAGUA. Cali. Colombia. 21 p.

UNIGARRO, A.; CARREÑO M. 2005. Métodos químicos para el análisis de suelo. Universidad de Nariño. Pasto, Colombia. $72 \mathrm{p}$.

ZAMORA, D. 2006. Cambios en algunas propiedades químicas del suelo según el uso de la tierra en el sector el Cebollal, estado Falcón, Venezuela. Revista Bioagro. 18(2):123 - 128 . 